International Journal of Case Reports
(ISSN:2572-8776)

\title{
Outcome of intrahepatic cholestasis of pregnancy: Case report
}

\author{
Avleen Kaur ${ }^{1}$, Gurinder Mohan ${ }^{2}$, Umang $^{3}$ \\ ${ }^{1}$ Medical officer, Singhania Hospital, Amritsar. \\ 2.Professor,Department of Medicine, Sri Guru Ram Das University of Health \& Science Amritsar, \\ Punjab, India. \\ ${ }^{3 .}$ Assistant Professor, Department of Obstetrics and Gynaecology, Sri Guru Ram Das University of \\ Health \& Sciences ,Amritsar, Punjab, India.
}

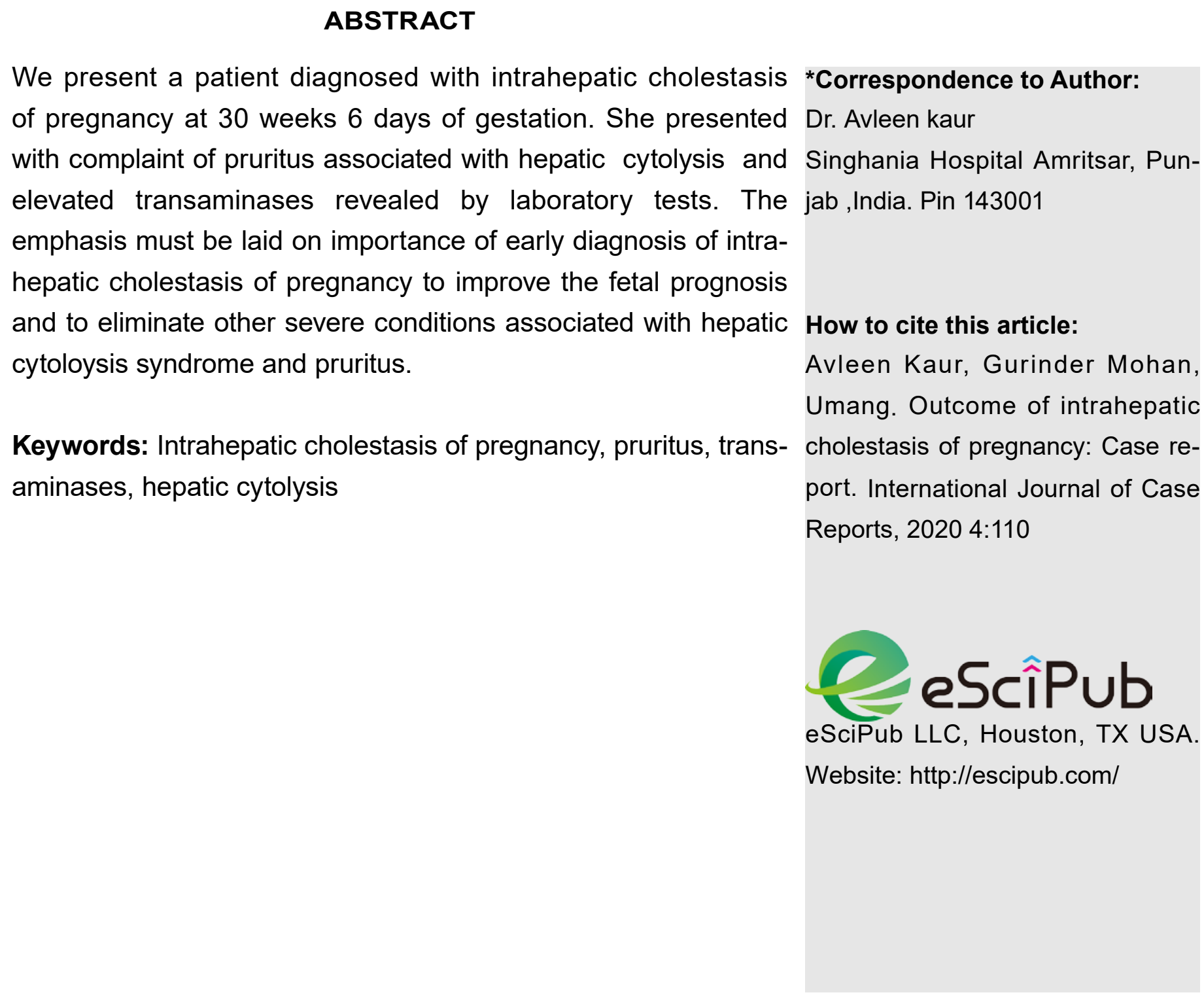




\section{INTRODUCTION:}

Intrahepatic cholestasis of pregnancy (ICP) has a potentially severe impact on maternal and fetal prognosis. Usually it appears in second and third trimester of pregnancy and is characterized by pruritus, elevation of transaminases, bilirubin levels, biliary enzymes and abnormal liver function tests ${ }^{1}$.The pruritus of obstetric cholestasis is typically worse at night, is often widespread and may involve palms and soles and it may precede the laboratory abnormalities. Pruritus can range in

severity from mild to moderate (where sleep is disrupted) and to extreme(when the lifestyle of the patient is completely disrupted).

The etiology of intrahepatic cholestasis of pregnancy is unknown, but genetic, hormonal and environmental factors are involved. ${ }^{2}$ The genetic variation around $A B C B 4$ and $A B C B 11$ encoding multidrug resistance protein(MDR3)and bile salt export pump(BSEP), respectively, have been reported as key factors related to etiology and severity of $\mathrm{ICP}^{3}$ Oestrogen is known to have role in cholestasis of pregnancy. Cholestasis is more common in twin pregnancies, which are associated with higher levels of circulating oestrogen than in singleton pregnancies. It can also be associated with altered metabolism of progesterone and progesterone administration may be a risk for cholestasis. ${ }^{4}$

The fetal consequences of ICP include spontaneous preterm labour, fetal distress and intrauterine death due to increased levels of serum bile acids $(>10 \mu \mathrm{mol} / \mathrm{L})$ which cause direct toxic effects on myocardium leading to arrhythmias. ${ }^{5}$ Early diagnosis and treatment with ursodeoxycholic acid (UDCA) and maturation of fetus lungs are necessary for both maternal and fetal outcome. Postnatal resolution of symptoms and biochemical abnormalities is required to confirm the diagnosis of intrahepatic cholestasis of pregnancy.

\section{CASE REPORT:}

A 25 years old patient, G2P1L0 with period of gestation 30 weeks 6 days was admitted in Obstetric and Gynaecology Department of Sri Guru Ram Das Hospital, Amritsar in Aug 2019. She complained of severe pruritus and jaundice. She had a similar episode in previous pregnancy and had intrauterine death at 28 weeks of gestation but it was not investigated.

Clinical examination was performed. Her blood pressure was normal i.e. $110 / 70 \mathrm{mmHg}$ and pulse rate was $70 \mathrm{bpm}$. She had mild icterus. No nausea, vomiting, headache or fatigue were noted. Laboratory investigations revealed increase in serum transaminases, alanine aminotransferase- $209.8 \mathrm{U} / \mathrm{L}$, aspartate transaminase $-282.2 \mathrm{U} / \mathrm{L}$, modestly increase in total bilirubin $-2.09 \mathrm{mg} / \mathrm{dL}$ and direct bilirubin $0.70 \mathrm{mg} / \mathrm{dl}$ (superior cut of values $1 \mathrm{mg} / \mathrm{dl}$ and $0.3 \mathrm{mg} / \mathrm{dl}$ respectively). Lactate dehydrogenase - $294 \mathrm{U} / \mathrm{L}$, alkaline phosphatase - $132.5 \mathrm{U} / \mathrm{L}$, total cholesterol - $246 \mathrm{mg} / \mathrm{dl}$, triglyceride -167 $\mathrm{mg} / \mathrm{dl}$. There was no proteinuria and coagulation profile were within normal limits. Her total bile acid levels were $19 \mu \mathrm{mol} / \mathrm{L}$ at the time of admission. No haemolysis or thrombocytopenia was observed and tests for viral markers including hepatitis $A, B$ and $C$ and $E$ were negative. Autoimmune markers including antinuclear, antimitochondral and antiphospholipid antibodies were negative. Based on laboratory investigations other liver disorders relating to pregnancy like viral hepatitis, chronic liver disease, HELLP syndrome, Acute fatty liver of pregnancy were excluded. The skin was inspected and other causes of pruritus like eczema, atopic eruptions, pruritic folliculitis were excluded.

After diagnosing intrahepatic cholestasis of pregnancy patient was treated with ursodeoxycholic acid (UDCA) $300 \mathrm{mg}$ twice a day and after two weeks levels of alamine aminotransferase increased to $290 \mathrm{U} / \mathrm{L}$ and aspartate transaminase increased to $424 \mathrm{U} / \mathrm{L}$ and alkaline phosphatase was raised to $188 \mathrm{U} / \mathrm{L}$ and her pruritus was not relieved and total bile acid levels were increased to $25 \mu \mathrm{mol} / \mathrm{L}$. The dose of UDCA was increased to $300 \mathrm{mg}$ thrice a 
day. Fetal monitoring was done by doing daily nonstress test, weekly biophysical profile and Doppler study. Within next two weeks pruritus was decreased, liver transaminases also showed decline, but bile acid levels further increased to $51.0 \mu \mathrm{mol} / \mathrm{L}$. At 35 weeks of gestation uterine contractions started spontaneously. Continuous electronic fetal monitoring revealed late decelerations and on amniotomy, meconium was confirmed and she underwent emergency caesarean section and a male baby $2000 \mathrm{gm}$ was delivered with Apgar score 7. The biochemical parameters normalized and pruritus disappeared within 2 weeks of delivery. The newborn had normal neuromotor and intellectual development.

\section{DISCUSSION}

The exact etiology of intrahepatic cholestasis is not known. There can be abnormal metabolism of bile acid due to high secretion of oestrogen during pregnancy. Various mutation of genes ABCB11, ABCB4 and ATP8B1 have been involved in development of ICP. ${ }^{6}$ These genes encode for proteins involved in transport of bile acids, phosphatidylcholine

and aminophospholipids. ${ }^{7}$ The patients who had taken micronised progesterones for preventing imminent premature labour, ICP was found in 64\% of cases. ${ }^{8}$ Serum cholesterol and triglycerides can also be elevated in patients of ICP. ${ }^{9}$

In our patient ICP was diagnosed at 30 weeks 6 days, in symptomatic context dominated by pruritus and was also associated with hepatic cytolysis syndrome and increased levels of bile acids at time of admission. We also considered eliminating other pathological conditions like pregnancy pruritus where tests show normal hepatic function and normal bile acids. Her serum markers showed no evidence of any other hepatic diseases, like HELLP syndrome, acute

fatty liver of pregnancy, autoimmune liver diseases or chronic hepatitis.

The most frequent complications in ICP to fetus is preterm delivery and the risk is higher if TBA $>40 \mu \mathrm{mol} / \mathrm{L}$ as found in our patient and she delivered at 35 weeks gestation. The cholelic acid activity result in increased sensitivity of uterine muscles to oxytocin and increased oxytocin receptor expression. There is increased incidence of intrapartum fetal distress or even intrauterine death. It can probably be due to direct toxic effects of bile acids upon the myocardium leading to arrhythmias. ${ }^{10}$ No correlation seems to exist between severity of maternal symptom and levels of bile acids. ${ }^{11}$

Administration of ursodeoxycholic acid has been encouraged for ICP to reduce pruritus and improve hepatobiliary enzyme levels and to reduce the risk of fetal death. ${ }^{12}$ In our case, UDCA was effective for improving symptoms. In some studies therapeutic use of dexamethasone and S.adenosyl-L-Methionine (SAME) has been described. In comparison to UDCA these therapies did not give better

outcomes with regard to reduction of laboratory parameters and clinical signs of cholestasis. ${ }^{13,14}$ Hydroxyzine $(25-50 \mathrm{mg}) \quad$ can alleviate pruritus but causes respiratory difficulty in premature infants. Cholestyramine (8$16 \mathrm{mg} /$ day) decreases the absorption of ileal bile salts and increase the fecal excretion. Its effect on pruritus is limited.

Pruritus disappears within first few days after delivery and there is normalization of serum bile acid concentrations and other liver function tests. McMenamin's study suggests that woman affected register an increase in liver sequelae, including gallstone disease, hepatitis, fibrosis and cholangitis. ${ }^{15}$ Biochemical liver tests and bile acid levels should be monitored six to eight weeks after delivery. If laboratory anomalies are not remitted, the patient should be referred to hepatologist to assess hepatobiliary disease.

\section{CONCLUSION:}

The patients with pruritus and abnormal serum transminases, especially in advanced stage of pregnancy can be having intrahepatic cholestasis of pregnancy. The affected pregnancies have increased risk of prematurity, fetal distress and intrauterine fetal death. The 
patient is treated with UDCA which lead to attenuation of pruritus and resolution of deranged biochemistry. UDCA normalises increased bile acid and reduces plasma concentration and urinary excretion rates of sulphate steroid metabolites. Intense monitoring of fetus status is mandatory. As there is risk of sudden intrauterine fetal death, pregnancy should be terminated after reaching the fetal lung maturity. Although treatment of ICP is not yet standardized, but it helps in improvement of fetal prognosis.

\section{REFERENCES:}

1. Chacko KR, Wolkoff AW. Intrahepatic cholestasis of pregnancy: new diagnostic insights. Annals of hepatology. 2017 Apr;16(2):176-8.

2. Arrese M, Macias RI, Briz O, Perez MJ, Marin JJ. Molecular pathogenesis of intrahepatic cholestasis of pregnancy. Expert reviews in molecular medicine. 2008 Mar;10.

3. Dixon PH, Wadswrth CA, ChambersJ, DonnellyJ,CooleyS,Buckley R,Mannino R,JarvisS,Syngelaki $\mathrm{P}$,SothinathanM, Kubitz A,GeenesV,Paul R,Lammert

F,TribeRM,Ch'ngCL,MarschallHU,GlantzA,Khan SA,NicolaidesK,WhittakerJ,GearyM,WilliamsonC: Acomprehensive analysis of common genetic variation Am J Gastroenterol 2014,109:76-84.

4. Meng LJ, Reyes H, Palma J, Hernandez I, Ribalta J, Sjövall J. Effects of ursodeoxycholic acid on conjugated bile acids and progesterone metabolites in serum and urine of patients with intrahepatic cholestasis of pregnancy. Journal of Hepatology. 1997 Dec 1;27(6):1029-40.

5. Williamson C, Gorelik J, Eaton BM, de swiet M, Korchev $Y$. The bile acid taurocholate impairs rat cardiomyocyte function: a proposed mechanism for intra-uterine fetal death in obstetric cholestasis. Clinical Science. 2001 Apr 1;100(4):363-9.

6. Müllenbach $R$, Bennett $A$, Tetlow $N$, Patel $N$, Hamilton G, Cheng F, Chambers J, Howard R, Taylor-Robinson SD, Williamson C. ATP8B1 mutations in British cases with intrahepatic cholestasis of pregnancy. Gut. 2005 Jun 1;54(6):829-34.

7. Van Mil SW, Milona A, Dixon PH, Mullenbach R, Geenes VL, Chambers J,Shevchuk V, Moore GE, Lammert F, Glantz AG, Mattsson LÅ. Functional variants of the central bile acid sensor FXR identified in intrahepatic cholestasis of pregnancy. Gastroenterology. 2007 Aug 1;133(2):507-16.

8. Bacq Y, Sapey T, Brechot M, Pierre F, Fignon A, Dubois $F$. Intrahepatic cholestasis of pregnancy: a French prospective study. Hepatology. 1997 Aug;26(2):358-64.

9. Reyes H,SjovallJ,.Bile acids and progesterone metabolites in intrahepatic cholestasis of pregnancy. Ann Med 2000;32:94-106.

10. Williamson C, Gorelik J, Eaton BM, de swiet M, Korchev $Y$. The bile acid taurocholate impairs rat cardiomyocyte function: a proposed mechanism for intra-uterine fetal death in obstetric cholestasis. Clinical Science. 2001 Apr 1;100(4):363-9.

11. Glantz A, Marschall HU, Mattsson LA. Intrahepatic cholestasis of pregnancy: relationships between bile acid levels and fetal complication rates. Hepatology. 2004 Aug;40(2):467-74.

12. Bacq $Y$, Sentilhes L, Reyes HB, Glantz A, Kondrackiene J, Binder T, Nicastri PL, Locatelli A, Floreani A, Hernandez I, Di Martino V. Efficacy of ursodeoxycholic acid in treating intrahepatic cholestasis of pregnancy: a meta-analysis. Gastroenterology. 2012 Dec 1;143(6):1492-501.

13. Diac M, Kenyon A, Nelson-Piercy C, Girling J, Cheng F, Tribe RM, Goodman J, Shennan A, Williamson C. Dexamethasone in the treatment of obstetric cholestasis: a case series. Journal of obstetrics and gynaecology. 2006 Jan ;26(2):1104.

14. Roncaglia N, Locatelli A, Arreghini A, Assi F, Cameroni I, Pezzullo JC, Ghidini A. A randomised controlled trial of ursodeoxycholic acid and Sadenosyl-I- methionine in the treatment of gestational cholestasis. BJOG: An International Journal of Obstetrics \& Gynaecology. 2004 Jan;111(1):17-21.

15. Marschael HV, Wikstrom Shemer E, Luvigsson JF, Stephansson O. Intrahepatic cholestasis of pregnancy and associated hepatobiliary disease: a population based cohort study. Hepatology $2013,58,1385$. 